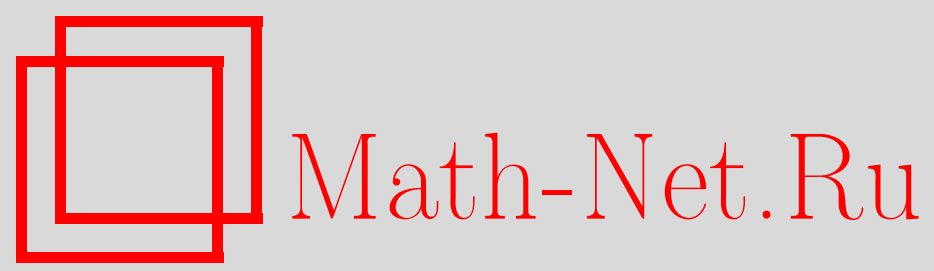

В. Б. Пеньков, И. Н. Стебенев, Метод состояний на основе уравнений Кильчевского для анализа трёхмерных установившихся колебаний, Вестн. Сам. гос. техн. ун-та. Сер. Физ.-мат. науки, 2011, выпуск 1(), 269-275

DOI: https://doi.org/10.14498/vsgtu864

Использование Общероссийского математического портала Math-Net.Ru подразумевает, что вы прочитали и согласны с пользовательским соглашением http://www.mathnet.ru/rus/agreement

Параметры загрузки:

IP : 54.162 .127 .20

26 апреля 2023 г., $16: 32: 41$ 
УДК 539.3

\section{МЕТОД СОСТОЯНИЙ НА ОСНОВЕ УРАВНЕНИЙ КИЛЬЧЕВСКОГО ДЛЯ АНАЛИЗА ТРЁХМЕРНЫХ УСТАНОВИВШИХСЯ КОЛЕБАНИЙ}

\section{В. Б. Пенъков, И. Н. Стебенев}

Липецкий государственный технический университет, 398600, Липецк, ул. Московская, 30.

E-mails: ViktorP@lipetsk.ru, Stebenev.Ivan@yandex.ru

Построено общее решение уравнений Н. А. Кильчевского для колеблющихся трехмерных тел. Обоснован метод состояний для анализа краевых задач о колебаниях, опирающийся на понятия: состояние среды (внутреннее, граничное), пространства состояний, скалярное произведение, гилъбертов изоморфизм.

Ключевые слова: теория упругости, общее решение, уравнения Кильчевского, метод граничных состояний, установившиеся колебания.

Введение. K настоящему времени возникла необходимость совершенствования существующих способов решения задач теории упругости в следующих направлениях: а) снижение уровня инструментальной ошибки; б) построение аналитического решения. Актуальным методом, отвечающим этим требованиям, является метод граничных состояний, изначально заявивший себя эффективным средством решения линейных задач механики сплошных сред [1]. Идеология метода граничных состояний ориентирована на символьное представление промежуточных и финишных результатов расчёта. Это отвечает современному уровню развития вычислительных средств, всё более ориентирующихся на компьютерные алгебры. Для многих прикладных задач заявленные к вычислению квадратуры берутся средствами компьютерной алгебры с абсолютной точностью.

Первоначально метод граничных состояний был основательно разработан для первой, второй, основной смешанной задач и основной контактной задачи линейной теории упругости. В дальнейшем метод граничных состояний получил своё развитие в применении к решению задач электростатического поля, идеальной и ньютоновской жидкости, а также статических задач неоднородной теории упругости, термоупругости и упругой анизотропии. Перспективным развитием является рассмотрение задач динамики (в частности, установившихся периодических движений).

Метод граничных состояний основан на понятии состояния среды, под которым понимается любое частное решение определяющих уравнений среды, построенное безотносительно к условиям на границе тела.

Внутреннее состояние $\xi=\left\{u_{j}, \varepsilon_{j m}, \sigma_{j m}\right\}$ упругой среды определяется наборами компонент вектора перемещений элемента среды $u_{j}$, компонент тензоров деформаций $\varepsilon_{j m}$ и напряжений $\sigma_{j m}$. Совокупность всех допустимых элементов $\xi$ образует пространство внутренних состояний $\Xi$.

Виктор Борисович Пеньков (д.ф.-м.н., проф.), профессор, каф. теоретической механики. Иван Николаевич Стебенев, аспирант, каф. теоретической механики. 
1. Общие уравнения Н. А. Кильчевского. Состояние движущейся изотропной линейно-упругой среды подчинено уравнениям движения, закону Гука (в форме Ламе) и соотношениям Коши. Н. А. Кильчевский [2] вывел более короткую форму представления состояния среды через «кинематические напряжения».

Компоненты тензора напряжений $\sigma_{j m}$ и вектора перемещений $u_{j}$ выражаются через три функции напряжения $\Psi_{1}, \Psi_{2}$ и $\Psi_{3}$ зависимостями (по повторяющимся индексам не суммировать)

$$
\begin{gathered}
\sigma_{j j}=\frac{1}{2}\left\{\frac{\partial^{2}}{\partial x_{l}^{2}}\left(\Psi_{j}+\Psi_{l}\right)+\frac{\partial^{2}}{\partial x_{m}^{2}}\left(\Psi_{m}+\Psi_{j}\right)-\frac{\rho}{\mu} \frac{\partial^{2}}{\partial t^{2}} \Psi_{j}\right\}, \\
\sigma_{m l}=-\frac{1}{2} \frac{\partial^{2}}{\partial x_{m} \partial x_{l}}\left(\Psi_{m}+\Psi_{l}\right), \quad u_{j}=-\frac{1}{2} \frac{\partial}{\partial x_{j}} \Psi_{j}, \quad j, m, l \in\{1,2,3\},
\end{gathered}
$$

где $\sigma_{j m}=\sigma_{m j}$-компоненты тензора напряжений; $x_{j}, x_{m}, x_{l}$-декартовы координаты; $\rho$-постоянная плотность среды; $\mu$-модуль сдвига; $t$-время.

Функции напряжений $\Psi_{j}$ подчинены системе из трёх дифференциальных уравнений в частных производных вида

$$
\Delta \Psi_{j}-\frac{\rho}{\mu} \frac{\partial^{2}}{\partial t^{2}} \Psi_{j}=-\frac{\lambda+\mu}{\mu}\left(\frac{\partial^{2}}{\partial x_{1}^{2}} \Psi_{1}+\frac{\partial^{2}}{\partial x_{2}^{2}} \Psi_{2}+\frac{\partial^{2}}{\partial x_{3}^{2}} \Psi_{3}\right),
$$

где $\Delta$ - оператор Лапласа; $\lambda$ - упругая постоянная Ламе.

Предположение о периодичности процессов во времени предопределяет пространственно-временную повторяемость в картине движения точек среды [3]:

$$
\exp \left[i\left(\vec{k} \cdot \vec{r}-s_{\eta} t\right) \omega / s_{\eta}\right], \quad \eta=1,2,
$$

где $i$-мнимая единица; $\vec{k}$-волновой вектор; $\vec{r}$ - радиус-вектор точки пространства; $\omega-$ круговая частота гармонических колебаний; $s_{\eta}-$ фазовая скорость.

Используя формулу Эйлера, представим выражение (2) в тригонометрическом виде, отделяя временной множитель $\exp (-i \omega t)$. Применим формулы сложения аргументов к полученным тригонометрическим выражениям. Пусть $\left\{\kappa_{d}\right\}_{d=0}^{\infty},\left\{\zeta_{w}\right\}_{w=0}^{\infty},\left\{\delta_{g}\right\}_{g=0}^{\infty}$ - последовательности положительных чисел, а системы элементов $\left\{\cos \left(\kappa_{d} x\right), \sin \left(\kappa_{d} x\right)\right\},\left\{\cos \left(\zeta_{w} y\right), \sin \left(\zeta_{w} y\right)\right\}$ и $\left\{\cos \left(\delta_{g} z\right)\right.$, $\left.\sin \left(\delta_{g} z\right)\right\}$ - базисы периодических функций от $x, y, z$. Их всевозможные произведения образуют базис троякопериодической функции (мономы) $X_{d}(x) Y_{w}(y) Z_{g}(z)$. Составляющие элементов базиса имеют следующие свойства:

$$
X_{d, x x}(x)=-\kappa_{d}^{2} X_{d}(x), \quad Y_{w, y y}(y)=-\zeta_{w}^{2} Y_{w}(y), \quad Z_{g}, z z(z)=-\delta_{g}^{2} Z_{g}(z) .
$$

Запятая в позиции нижних индексов говорит о дифференцировании по последующим индексам.

Разложим функции Н. А. Кильчевского $\Psi_{j}$ в периодические функции времени $\Psi_{j}=\Psi_{j}^{*} \exp (i \omega t)\left(\Psi_{j}^{*}\right.$ не зависит от времени). Примем содержательные обозначения $x_{1}=x, x_{2}=y, x_{3}=z, \Psi_{1}=\Phi, \Psi_{2}=\Psi, \Psi_{3}=\mathrm{H}$ и перепишем 
(1), раскладывая искомые функции в тригонометрические ряды. Приходим к системе

$$
\begin{aligned}
& (1+\beta) \Phi_{, x x}^{*}+\Phi_{, y y}^{*}+\Phi_{, z z}^{*}+\chi^{2} \Phi^{*}+\beta\left(\Psi_{, y y}^{*}+\mathrm{H}_{, z z}^{*}\right)=0 \\
& \Psi_{, x x}^{*}+(1+\beta) \Psi_{, y y}^{*}+\Psi_{,{ }_{z z}}^{*}+\chi^{2} \Psi^{*}+\beta\left(\Phi_{, x x}^{*}+\mathrm{H}_{, z z}^{*}\right)=0, \\
& \mathrm{H}_{, x x}^{*}+\mathrm{H}_{, y y}^{*}+(1+\beta) \mathrm{H}_{, z z}^{*}+\chi^{2} \mathrm{H}^{*}+\beta\left(\Phi_{, x x}^{*}+\Psi_{, y y}^{*}\right)=0,
\end{aligned}
$$

где $\beta=(\lambda+\mu) / \mu ; \chi=\omega / s_{2}-$ волновое число поперечных колебаний, $s_{2}=$ $=\sqrt{\mu / \rho}-$ фазовая скорость поперечных колебаний.

Будем искать разложения функций формы в виде рядов

$$
\left\{\Phi^{*}, \Psi^{*}, \mathrm{H}^{*}(x, y, z)\right\}=\sum_{d} \sum_{w} \sum_{g}\left\{a_{d w g}, b_{d w g}, c_{d w g}\right\} X_{d}(x) Y_{w}(y) Z_{g}(z),
$$

где $a_{d w g}, b_{d w g}, c_{d w g}$ - коэффициенты разложения функций формы.

При учёте линейной независимости $X_{d}(x) Y_{w}(y) Z_{g}(z)$ подстановка (4) в (3) приводит к уравнению относительно $a, b, c$ (индексы $d, w, g$ опускаем):

$$
\left(\begin{array}{ccc}
q-\beta \kappa^{2} & -\beta \zeta^{2} & -\beta \delta^{2} \\
-\beta \kappa^{2} & q-\beta \zeta^{2} & -\beta \delta^{2} \\
-\beta \kappa^{2} & -\beta \zeta^{2} & q-\beta \delta^{2}
\end{array}\right)\left(\begin{array}{l}
a \\
b \\
c
\end{array}\right)=\left(\begin{array}{l}
0 \\
0 \\
0
\end{array}\right)
$$

где $q=\chi^{2}-\left(\zeta^{2}+\delta^{2}+\kappa^{2}\right)$.

Уравнение (5) имеет два действительных нетривиальных решения, которые согласуют функции формы $\Psi_{j}^{*}(x, y, z)$ :

$$
\kappa^{2}+\zeta^{2}+\delta^{2}=\chi^{2}, \quad \kappa_{0}^{2}+\zeta_{0}^{2}+\delta_{0}^{2}=\chi_{0}^{2},
$$

где $\chi_{0}^{2}=\chi^{2} /(1+\beta)=\omega^{2} / s_{1}^{2} ; s_{1}^{2}=(\lambda+2 \mu) / \rho$ - фазовая скорость продольных колебаний.

Тогда общее решение уравнения (5) выражается в виде

$$
\left(\begin{array}{l}
a \\
b \\
c
\end{array}\right)=C_{1}\left(\begin{array}{c}
-(\zeta / \kappa)^{2} \\
1 \\
0
\end{array}\right)+C_{2}\left(\begin{array}{c}
-(\delta / \kappa)^{2} \\
0 \\
1
\end{array}\right)+C_{3}\left(\begin{array}{l}
1 \\
1 \\
1
\end{array}\right)
$$

где $C_{1}, C_{2}$ и $C_{3}$ - произвольные постоянные.

Первое слагаемое в (7) определяет плоскую гармоническую горизонтально поляризованную волну сдвига, второе - плоскую гармоническую вертикально поляризованную волну сдвига, третье - продольную волну.

Каждая тройка индексов порождает три набора из функций формы, удовлетворяющих уравнениям (4) и (5):

$$
\left(\begin{array}{l}
\Phi_{d w g}^{*}(x, y, z) \\
\Psi_{d w g}^{*}(x, y, z) \\
\mathrm{H}_{d w g}^{*}(x, y, z)
\end{array}\right) \in\left\{\left(\begin{array}{c}
a_{d w g} \\
b_{d w g} \\
0
\end{array}\right),\left(\begin{array}{c}
a_{d w g} \\
0 \\
c_{d w g}
\end{array}\right),\left(\begin{array}{l}
1 \\
1 \\
1
\end{array}\right)\right\} X_{d}(x) Y_{w}(y) Z_{g}(z)
$$

С учётом (6) представим последовательность чисел $\left\{\kappa_{d}\right\},\left\{\zeta_{w}\right\},\left\{\delta_{g}\right\}$ в качестве узловых точек в трёхмерной прямоугольной Гауссовской сетке, оси которой ориентированы по нормали к волновому фронту:

$$
\kappa=\chi \cos (\varphi) \cos (\theta), \quad \zeta=\chi \sin (\varphi) \cos (\theta), \quad \delta=\chi \sin (\theta)
$$




$$
\kappa_{0}=\chi_{0} \cos (\varphi) \cos (\theta), \quad \zeta_{0}=\chi_{0} \sin (\varphi) \cos (\theta), \quad \delta_{0}=\chi_{0} \sin (\theta),
$$

где $\varphi \in[0 ; \pi / 2)$ - долгота; $\theta \in[0 ; \pi / 2)$ - широта.

Совокупность вышеприведённых соотношений даёт общие решения уравнений Н. А. Кильчевского. Н. И. Остросаблин [4] вывел более общий способ задания функций кинематических напряжений, для которого уравнения Н. А. Кильчевского являются частным случаем.

2. Теоретическое обоснование метода состояний. Применим решение Н. А. Кильчевского для определения основных характеристик среды. Через полученные разрешающие соотношения для периодических колебаний упругих тел «набирается» сепарабельный базис $\xi$ пространств внутренних состояний $\Xi$.

Для двух произвольных состояний $\xi^{(1)}$ и $\xi^{(2)}$ вводится скалярное произведение, определяемое через интеграл от свёртки тензоров напряжений и деформаций по занятой телом области $V$ :

$$
\left(\xi^{(1)}, \xi^{(2)}\right)_{\Xi}=\int_{V} \sigma_{j m}^{(1)} \varepsilon_{j m}^{(2)} d V
$$

Выполняется ортогонализация исходного базиса (применяется рекурсивный матричный алгоритм [5]).

На границе $\partial V$ тела внутреннее состояние $\xi$ оставляет «след» в виде поверхностных усилий $p_{j}$, которые вместе с граничными значениями перемещения $u_{j}$ образуют граничное состояние $\gamma=\left\{u_{j}, p_{j}\right\}$. Строится базис элементов пространства граничных состояний $\Gamma$, ортогональный в смысле скалярного произведения - поверхностного интеграла от свёртки векторов поверхностных усилий и перемещений. В пространстве граничных состояний скалярное произведение определим так:

$$
\left(\gamma^{(1)}, \gamma^{(2)}\right)_{\Gamma}=\int_{\partial V} p_{j}^{(1)} u_{j}^{(2)} d S-\rho \int_{V} \ddot{u}_{j}^{(1)} u_{j}^{(2)} d V
$$

где $d S$ - элемент поверхности; $\ddot{u}_{j}$ - компоненты вектора ускорения элемента среды.

Постольку по граничному состоянию $\gamma$ можно единственным образом восстановить внутреннее $\xi$ (формулы Сомильяны), то между пространствами $\Xi$ и $Г$ установлено взаимнооднозначное соответствие: $\Xi \leftrightarrow \Gamma$. В силу теоремы Бетти и принципа виртуальных работ [6] оба пространства внутренних и граничных состояний $\Xi$ и $Г$ сопряжены гильбертовым изоморфизмом:

$$
\xi^{(1)}+\xi^{(2)} \leftrightarrow \gamma^{(1)}+\gamma^{(2)}, \quad \nu \xi \leftrightarrow \nu \gamma, \quad \nu \in \mathrm{R}^{1} ; \quad\left(\xi^{(1)}, \xi^{(2)}\right)_{\Xi}=\left(\gamma^{(1)}, \gamma^{(2)}\right)_{\Gamma} .
$$

Это позволяет задачу отыскания внутреннего состояния свести к проблеме построения изоморфного ему граничного состояния, которое существенно зависит от краевых условий. В общем случае проблема сводится к решению системы уравнений разложением относительно коэффициентов Фурье $c_{h}$ искомых состояний в ряд по элементам ортонормированного базиса:

$$
p_{j}=\sum_{h=1}^{\infty} c_{h} p_{j}^{(h)}, \quad u_{j}=\sum_{h=1}^{\infty} c_{h} u_{j}^{(h)}, \quad \varepsilon_{j m}=\sum_{h=1}^{\infty} c_{h} \varepsilon_{j m}^{(h)}, \quad \sigma_{j m}=\sum_{h=1}^{\infty} c_{h} \sigma_{j m}^{(h)} .
$$


Определение коэффициентов Фурье при ортонормированных элементах $\gamma^{(n)}$ базиса пространства $\Gamma$ таково:

- в первой основной задаче:

$$
c_{n}=\left(\gamma, \gamma^{(n)}\right)=\left.\int_{\partial V} p_{j} u_{j}^{(n)}\right|_{\partial V} d S-\rho \int_{V} \ddot{u}_{j} u_{j}^{(n)} d V
$$

- во второй основной задаче:

$$
c_{n}=\left(\gamma, \gamma^{(n)}\right)=\left.\int_{\partial V} p_{j}^{(n)}\right|_{\partial V} u_{j} d S-\rho \int_{V} \ddot{u}_{j} u_{j}^{(n)} d V .
$$

Соотношения (8) и (9) приводят к бесконечной системе линейных алгебраических уравнений

$$
\sum_{h=1}^{\infty}\left(\delta_{n h}+a_{n h}\right) c_{h}=b_{n}, \quad a_{n h}=\rho \int_{V} \ddot{u}_{j}^{(h)} u_{j}^{(n)} d V,
$$

где $\delta_{n h}-$ символ Кронекера; $b_{n}=\left.\int_{\partial V} p_{j} u_{j}^{(n)}\right|_{\partial V} d S$ - для первой основной задачи; $b_{n}=\left.\int_{\partial V} p_{j}^{(n)}\right|_{\partial V} u_{j} d S$ - для второй основной задачи.

3. Тестовая задача. Рассматривается тело из изотропного материала, ограниченное двумя конусами с общим радиусом основания $R=1$ и такой же высотой (ниже - «конусное тело», рис. 1). Используются безразмерные величины: параметры Ламе $(\lambda=\mu=1)$, плотность $\rho=1$; тело колеблется с частотой $\omega=1$.

Рассматривается первая основная задача: на поверхность «конусного тела» действует сдавливающая нагрузка в проекциях на оси $x, y, z$ (рис. 1$)$ :

$$
\begin{gathered}
\left.Q\right|_{S_{1}}=\{-\cos (\alpha) / \sqrt{2} ;-\sin (\alpha) / \sqrt{2} ; 1 / \sqrt{2}\}, \\
\left.Q\right|_{S_{2}}=\{0 ; 0 ;-1 / \sqrt{2}\}
\end{gathered}
$$

где $Q$-периодические поверхностные силы; $S_{1}, S_{2}$ - нижняя и верхняя конусная поверхность тела соответственно; $\alpha \in[-\pi ; \pi]$.

Построены пространственные графики зависимостей исследуемых характеристик от радиальной координаты $R$ и

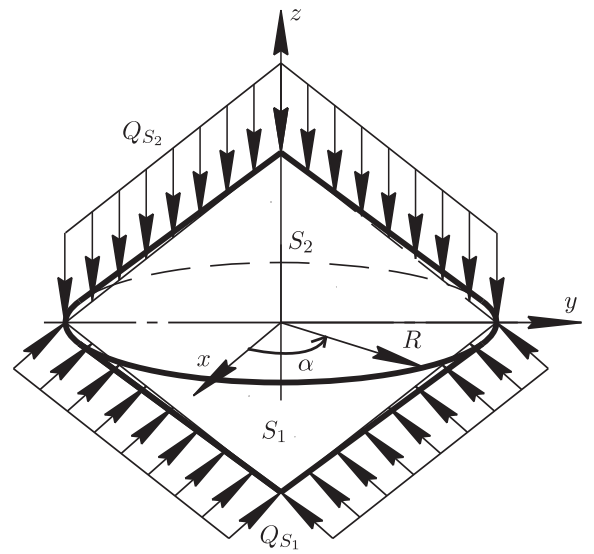

Рис. 1. «Конусное тело» полярного угла $\alpha$. Сравниваются осевые усилия $P_{Z}$ (графики слева) и радиальные нагрузки $P_{R}$ (изображения справа):

a) усилия $Q$ (светлая поверхность), б) поверхностные силы $p_{j}$, полученные при расчётах вышеописанным методом (тёмная поверхность) (рис. 2, 3). 

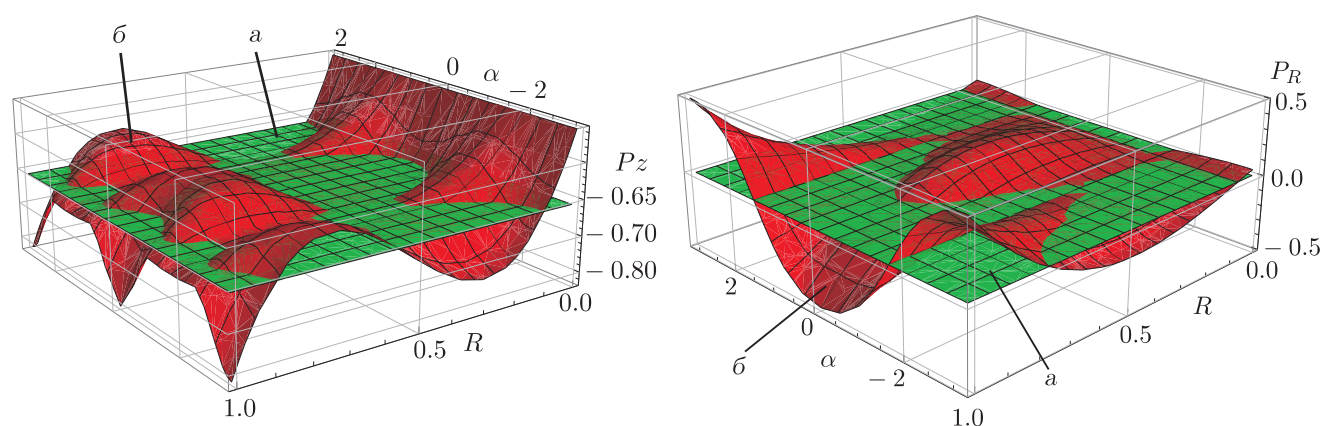

Рис. 2. Усилия на верхней поверхности «конусного тела»

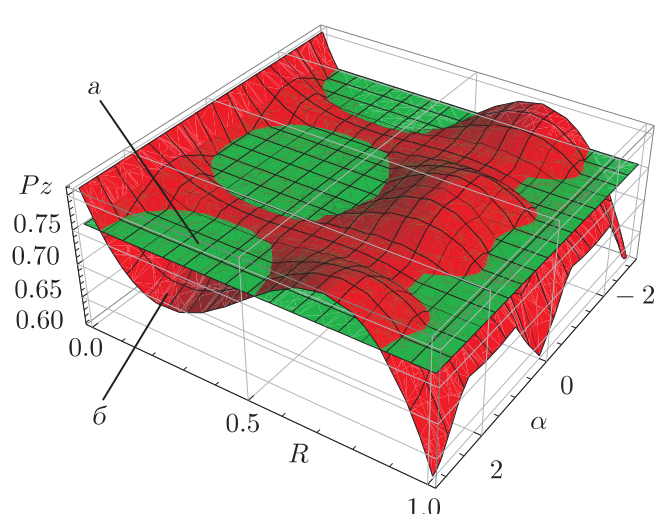

1.0

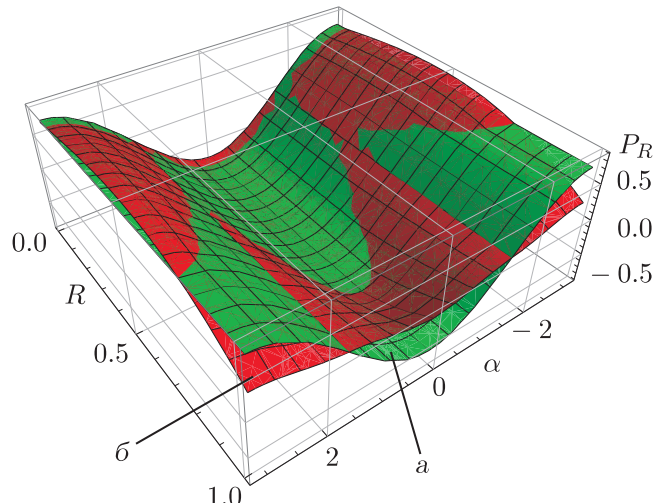

Рис. 3. Усилия на нижней поверхности «конусного тела»

Заключение. Решены первая и вторая основные задачи об установившихся колебаниях для односвязных изотропных упругих тел. Результаты решения поставленных задач имеют аналитическую форму, что позволяет легко их анализировать. Разработана система жесткого тестирования промежуточных и окончательных результатов решения задач. Для снижения погрешности вычислений рекомендуется наращивать количество используемых элементов базисов пространств состояний.

Работа выполнена в рамках тематического плана ГОУ ВПО «ЛГТУ» по заданию Минорбнауки РФ (№ НИР 1.3.11).

\section{БИБЛИОГРАФИЧЕСКИЙ СПИСОК}

1. Пенъков В.Б., Пенъков В.В. Метод граничных состояний для решения задач линейной механики // Дальневост. матем. журн., 2001. Т. 2, № 2. С. 115-137. [Pen’kov V. B., Pen'kov V.V. Boundary conditions method for solving linear mechanics problems // Dal'nevost. matem. zhurn., 2001. Vol. 2, no. 2. Pp. 115-137].

2. Килъчевский Н. А. Основы тензорного исчисления с приложениями к механике. Киев: Наукова думка, 1972. 148 с. [Kil'chevskiy N. A. The foundations of the tensor calculus, with applications to mechanics. Kiev: Naukova dumka, 1972. 148 pp.]

3. Гринченко В. T., Мелешко В.В. Гармонические колебания и волны в упругих телах. Киев: Наукова думка, 1981. 284 с. [Grinchenko V. T., Meleshko V. V. Harmonic oscillations and waves in elastic bodies. Kiev: Naukova dumka, 1981. 284 pp.]

4. Остросаблин Н.И. Функции кинетических напряжений в механике сплошных сред/ 
Динамика сплошной среды. Новосибирск, 2007. С. 76-116. [Ostrosablin N. I. Functions of kinetic stresses in continuum mechanics / Dinamika sploshnoy sredy, 2007. Pp. 76-116].

5. Пенъков В.Б., Саталкина Л.В. Эффективные алгоритмы метода граничных состояний // Вестн. ТулГУ. Сер. Актуалъные вопросы механики, 2010. №2. С. 91-96. [Pen'kov V.B., Satalkina L. V. Efficient algorithms for the method of boundary states // Vestn. TulGU. Ser. Aktual'nye voprosy mekhaniki, 2010. no. 2. Pp. 91-96].

6. Горшков А.Г., Старовойтов Э.И., Тарлаковский Д. В. Теория упругости и пластичности. М.: Физматлит, 2002. 416 с. [Gorshkov A. G., Starovoytov E.I, Tarlakovsky D. V. Theory of elasticity and plasticity. Moscow: Fizmatlit, 2002. 416 pp.]

MSC: 74B05

\section{METHOD OF STATES ON THE BASIS OF KILCHEVSKIY'S EQUATIONS FOR ANALYSIS OF 3D STEADY-STATE OSCILLATION}

\section{V.B. Pen'kov, I. N. Stebenev}

Lipetsk State Technological University,

30, Moskovskaya st., Lipetsk, 398600, Russia.

E-mails: ViktorP@lipetsk.ru, Stebenev.Ivan@yandex.ru

General solution of N. A. Kilchevskiy's equations for oscillating three-dimensional solids is constructed. Method of states for analysis of boundary-value problem about oscillations is proven, it is based on the following terms: states of medium (internal and boundary), space of states, scalar product, gilbert isomorphism.

Key words: theory of elasticity, general solution, Kilchevskiy's equations, method of boundary states, steady-state oscillation.

Original article submitted 20/XII/2010; revision submitted $22 / \mathrm{II} / 2011$.

Victor B. Pen'kov (Dr. Sc. (Phys. \& Math.)), Professor, Dept. of Theoretical Mechanics. Ivan N. Stebenev (Postgraduate Student), Dept. of Theoretical Mechanics. 